\title{
Research on Precise Modeling and Its Robust Control Strategy of Shunt Active Power Filter
}

\author{
Zhan Shu ${ }^{a}$, Xia Cai ${ }^{b}$, Qiong Wang ${ }^{c}$, Bo Chen ${ }^{d}$, Ning Zhou ${ }^{e}$ and Liang Tang ${ }^{f}$ \\ Electric Power Research Institute of Jiangxi Electricity Company of State Grid, Nanchang City 330096, \\ China \\ ashuzhan2003@126.com, bjjcaixia@126.com, c365936179@qq.com, d19998933@qq.com, \\ ezhouning1982@163.com, 'suyongchun@126.com
}

Keywords: Shunt active power filter; repetitive control; passive control; robust current control.

\begin{abstract}
Shunt active power filter (APF) has become the most effective tool for harmonic control in the industrial field because of its excellent performance. But in the field operation of APF is very complex, all kinds of interference factors of certainty and uncertainty always exist, the reliable operation of the device has a very serious harm. In this paper, an accurate mathematical model of APF in abc coordinates is established; By means of equivalent transformation, the parameters perturbation of the model are transformed into a part of periodic disturbance; the robust current control of APF is realized by the combination of repetitive control and passive control, the repetitive control compensates period disturbance, the passive control ensures the convergence of the repetitive control, and the effect of the aperiodic disturbance is suppressed. The accuracy of the mathematical model is verified by the simulation and engineering application. The control algorithm is simple and the practical engineering value is high.
\end{abstract}

\section{Introduction}

With the rapid development of modern industrial technology, the wide use of various non-linear load in electric system, with power electronic device as a typical example, has made harmonic in public power grid become one of the main problems of the power supply quality of electric system. The shunt active power filter mainly uses power electronic devices (eg. MOSFET, IGBT, etc.) to make up a self-commutated convertor, and offers harmonic current through the invertion technology of voltage source required by load. Compared with the traditional passive power filter, with many advantages such as rapid response speed, automatic tracking of the change of power grid frequency, not easy syntony with power grid, one-time centralized compensation, compensation of harmonic wave and reactive power at the same time, without influence of system voltage and frequency change on compensation characteristic, APF is the most effective tool for current harmonic suppression [1-4].

APF is a typical nonlinear system, with very complex operation condition. There are always the phenomenon of component parameter perturbation, the dead zone effect of inverter, pulse delay and compensatory static errors, as well as load fluctuation, system fluctuation, pulse loss and other uncertain factors, with very serious influence on the control effect of device [5]. Therefore, to build APF, we shall consider the unknown disturbance and the precise mathematical model of parameter perturbation, analyze its influence on the control performance of device, and guarantee the safe, reliable and stable operation of device under the complex operating condition, which are difficulty in research for APF, but few literatures discuss on it at present.

Based on the repetitive control of internal model principle, the gradual pointed compensation with the application of the periodic law of error signal, we can eliminate all periodic errors within closed-loop system, make systemic periodic errors reduced step by step or eliminated, thus make the system output achieve expected goal [6-8]. The passive control theory is an important tool to design the robust equanimity controller in nonlinear system, which is a kind of control method for energy shaping. By the infusion of nonlinear damping, it can compel the gross energy of system to track the expected energy function, and force the state variable of system to asymptotically converge to the set 
value and attain the performance [9-11] required by the system. This paper uses a new method with combination of repetitive control and passive control to realize the robust current controller design [12-15] of APF, among which repetitive control compensates for periodic disturbance, passive control guarantees the astringency of repetitive control and asymptotic tracking of the control objective, and restrains the influence of non-periodic disturbance on control effect. This method has overall definition and is stable in whole, without singular point and with simple design of controller. As shown in simulation and experiment, this control algorithm can control harmonic current, with good steady-state characteristic, rapid dynamic response, simple algorithm implementation, strong robustness and very strong engineering practicability.

\section{System modeling}

The main circuit topology of APF is shown as Fig.1. In the figure, PCC (point of common coupling) is the point of junction for APF to connect with electric system. If setting the detection point of system voltage to be PCC, according to circuit substitution theorem, the system impedance can be not considered in the equivalent circuit. In Fig. $1, u_{s x}(x=a, b, \mathrm{c})$ is three-phase system voltage; $i_{s x}$ is three-phase system current; $i_{c x}$ is the offset current of device; $i_{l x}$ is the current of load side; $L_{s x}$ is linked electric reactor; device loss (including the internal series connection loss of inverter itself and the loss of linked electric reactor) is represented by equivalent series resistance $R_{s x}$; the parallel connection loss at the direct current side of inverter is represented by equivalent conductance $Y_{d} ; C_{d}$ is the capacitance at the direct current side of inverter; $u_{d}$ is the capacitor voltage at the direct current side of inverter. In reality, the control objective of APF is to require the harmonic component in the current at system side $i_{s x}$ to be equal to zero at the same time when the capacitor voltage at the direct current side of inverter is stable to be the reference value.

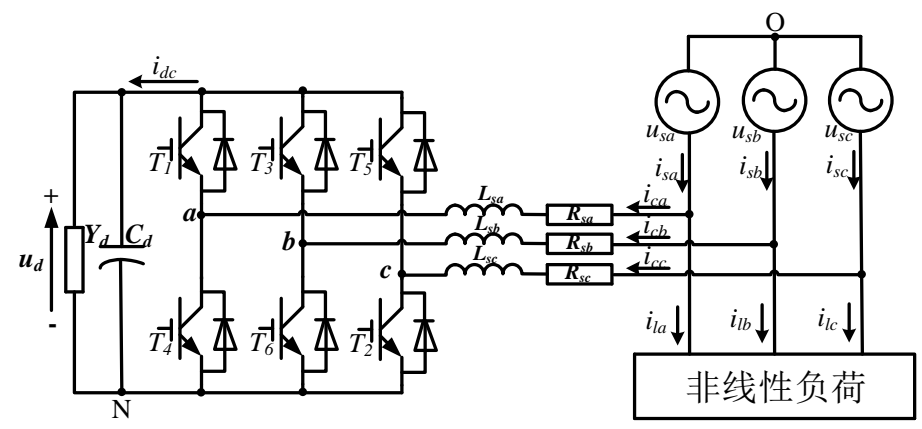

Fig. 1 Main circuit topology of shunt active power filter

Suppose the duty ratio function of APF is $p_{x}(x=a, b, c)$. Based on no access of zero-sequence component in three-phase three-wire system circuit, in accordance with Kirchhoff Voltage Law, we can get that, the state average model of APF shown in Fig. 1 is

$$
\left\{\begin{array}{l}
L_{s a} \frac{\mathrm{d} i_{c a}}{\mathrm{~d} t}+p_{a} u_{d}+R_{s a} i_{c a}=u_{s a} \\
L_{s b} \frac{\mathrm{d} i_{c b}}{\mathrm{~d} t}+p_{b} u_{d}+R_{s b} i_{c b}=u_{s b} \\
L_{s c} \frac{\mathrm{d} i_{c c}}{\mathrm{~d} t}+p_{c} u_{d}+R_{s c} i_{c c}=u_{s c} \\
C_{d} \frac{d u_{d}}{d t}-\left(p_{a} i_{c a}+p_{b} i_{c b}+p_{c} i_{c c}\right)+Y_{d} u_{d}=0
\end{array}\right.
$$

APF is a nonlinear system, with very complex operation condition, which will suffer various unknown disturbances in actual operation. The unknown disturbance $w_{k}(k=a, b, c, d)$ is usually bounded and $w_{k} \in L_{2}[0, \mathrm{~T}]$. Meanwhile, the phenomenon of component parameter perturbation is also universal, all true values of $L_{s x}, R_{s x}$ and $Y_{\mathrm{d}}$ can't be precisely confirmed, and the capacity of Capacitance $C_{\mathrm{d}}$ will also reduce slowly with the ageing of device. Suppose $L_{s x}, R_{s x}, C_{\mathrm{d}}$ and $Y_{\mathrm{d}}$ can be respectively represented as 


$$
\left\{\begin{array}{l}
L_{s x}=L+\Delta L_{x} \\
R_{s x}=R+\Delta R_{x} \\
C_{d}=C+\Delta C \\
Y_{\mathrm{d}}=Y+\Delta Y
\end{array}\right.
$$

In the formula: $L, R, C$ and $Y$ are all known fixed constants; $\Delta L_{x}, \Delta R_{x}, \Delta C$ and $\Delta Y$ are corresponding parameter perturbation items.

In connection between (1) and (2), we can get that,

$$
\left\{\begin{array}{l}
\left(L+\Delta L_{a}\right) \frac{d i_{c a}}{d t}+p_{a} u_{d}+\left(R+\Delta R_{a}\right) i_{c a}=u_{s a}+w_{a} \\
\left(L+\Delta L_{b}\right) \frac{d i_{c b}}{d t}+p_{b} u_{d}+\left(R+\Delta R_{b}\right) i_{c b}=u_{s b}+w_{b} \\
\left(L+\Delta L_{c}\right) \frac{d i_{c c}}{d t}+p_{c} u_{d}+\left(R+\Delta R_{c}\right) i_{c c}=u_{s c}+w_{c} \\
(C+\Delta C) \frac{d u_{d}}{d t}-p_{a} i_{c a}-p_{b} i_{c b}-p_{c} i_{c c}+(Y+\Delta Y) u_{d}=w_{d}
\end{array}\right.
$$

Formula (3) is just the precise mathematical model of Shunt APF in $a b c$ static coordinate system which takes the influence of unknown disturbance and parameter perturbation into account. In actual operation, $w_{k}(k=a, b, c, d)$ is mainly from the dead zone effect of inverter, pulse delay and compensatory static errors and other periodic disturbances, as well as load fluctuation, system fluctuation, pulse loss and other non-periodic disturbances.

Suppose the control objective of APF is $\mathrm{x}^{*}=\left[\mathrm{ica}^{*}, \mathrm{icb}^{*}, \mathrm{icc}^{*}, \mathrm{ud}^{*}\right]$, among which icx* is directive value of the offset current of device, $\mathrm{ud}^{*}$ is directive voltage of capacitance at the direct current side of inverter.

Define the systematic state variable as

$$
z_{e}=\left[\begin{array}{c}
z_{e a} \\
z_{e b} \\
z_{e c} \\
z_{e d}
\end{array}\right]=\left[\begin{array}{c}
i_{c a}-i_{c a}^{*} \\
i_{c b}-i_{c b}^{*} \\
i_{c c}-i_{c c}^{*} \\
u_{d}-u_{d}^{*}
\end{array}\right]
$$

Substitute Formula (4) into Formula (3), taking APF error system model of the unknown disturbance and parameter perturbation influence into account:

$$
N \frac{d z_{e}}{d t}+J z_{e}+R z_{e}=\xi-\Delta N \frac{d x^{*}}{d t}-\Delta R x^{*}+w
$$

In the formula: $N=\operatorname{diag}\left(L+\Delta L_{a}, L+\Delta L_{b}, L+\Delta L_{c}, C+\Delta C\right)$ is a symmetry positive definite matrix; $\boldsymbol{J}=\left[\begin{array}{cccc}0 & 0 & 0 & p_{a} \\ 0 & 0 & 0 & p_{b} \\ 0 & 0 & 0 & p_{c} \\ -p_{a} & -p_{b} & -p_{c} & 0\end{array}\right]$ is inside the system; $R=\operatorname{diag}\left(R+\Delta R_{a}, R+\Delta R_{b}, R+\Delta R_{c}, Y+\Delta Y\right)$ is a symmetrical positive definite matrix, which reflects the dissipation characteristic of system; $\boldsymbol{\xi}=\boldsymbol{u}_{s^{-}}\left(\boldsymbol{N} \mathbf{1} \boldsymbol{d} \boldsymbol{x}^{*} / \boldsymbol{d} \boldsymbol{t}+\boldsymbol{J} \boldsymbol{x}^{*}+\boldsymbol{R} \mathbf{1} \boldsymbol{x}^{*}\right), \boldsymbol{u}_{s}=\left[\boldsymbol{u}_{s a}, \boldsymbol{u}_{s b}, u_{s c}, 0\right]^{\mathrm{T}}$, $N_{\mathbf{1}}=\operatorname{diag}(L, L, L, C), \boldsymbol{R} \mathbf{1}=\operatorname{diag}(R, R, R, Y) ; \Delta \boldsymbol{N}=\operatorname{diag}\left(\Delta L_{a}, \Delta L_{b}, \Delta L_{c}, \Delta C\right) ; \Delta \boldsymbol{R}=\operatorname{diag}\left(\Delta R_{a}, \Delta R_{b}, \Delta R_{c}, \Delta Y\right)$; $\boldsymbol{w}=\left[w_{a}, w_{b}, w_{c}, w_{d}\right]^{\mathrm{T}}$.

The bounded unknown disturbance variable $w_{k}(k=a, b, c, d)$ can be further represented as wk=wdk+wlk

In the formula: $w_{d k}$ is periodic disturbance, and can satisfy $w_{d k}(t)=w_{d k}\left(t-T_{f}\right)(k=a, b, c, d), T_{f}$ is power frequency cycle; $w_{l k}$ is non-periodic disturbance. Correspondingly, $w$ can be also resolved into two parts, periodic disturbance vector and non-periodic disturbance vector:

$\mathrm{w}=\mathrm{wd}+\mathrm{wl}$

In the formula: $\boldsymbol{w}_{d}=\left[w_{d a}, w_{d b}, w_{d c}, w_{d d}\right]^{\mathrm{T}} ; \boldsymbol{w} l=\left[w_{l a}, w_{l b}, w_{l c}, w_{l d}\right]^{\mathrm{T}}$. 
In Formula (5), $\Delta \boldsymbol{N} \boldsymbol{d} \boldsymbol{x}^{*} / \boldsymbol{d t}, \Delta \boldsymbol{R} \boldsymbol{x}^{*}$ are also periodic function vectors. Let $\boldsymbol{w}_{p}=-\Delta \boldsymbol{N} \boldsymbol{d} \boldsymbol{x} * \boldsymbol{d} \boldsymbol{t}-\Delta \boldsymbol{R} \boldsymbol{x}^{*}+\boldsymbol{w}_{\mathrm{d}}=\left[w_{p a}, w_{p b}, w_{p c}, w_{p d}\right]^{\mathrm{T}}$. Obviously, $\boldsymbol{w}_{\boldsymbol{p}}$ is also a function vector with $T_{f}$ as its cycle, then the error system model shown by Formula (5) can be simplified as

$$
N \frac{d z_{e}}{d t}+J z_{e}+R z_{e}=\xi+w_{p}+w_{l}
$$

As shown in Formula (8), in the formula (5), convert the influence of component parameter perturbation into a part of periodic disturbance via equivalence transformation, which can avoid using self-adaptive identification to carry out special compensation to component parameter perturbation.

\section{Robust current control}

\subsection{Repetitive control}

In actual operation obviously, $w_{p a} \in L_{\infty}, w_{p b} \in L_{\infty}, w_{p c} \in L_{\infty}, w_{p d} \in L_{\infty}$. Define $\hat{\boldsymbol{w}}_{p}=\left[\hat{w}_{p a}, \hat{w}_{p b}, \hat{w}_{p c}, \hat{w}_{p d}\right]^{\mathrm{T}}$ as the estimated vector of $\boldsymbol{w}_{\boldsymbol{p}}$. Suppose the energy storage function of error system equation (8) is

$$
V(t)=\frac{1}{2} z_{e}^{T} N z_{e}+\frac{1}{2 q} \int_{t-T_{f}}^{t}\left\|\boldsymbol{w}_{p}(\tau)-\hat{\boldsymbol{w}}_{p}(\tau)\right\|^{2} \mathrm{~d} \tau
$$

In the formula:\|.\| is the Euclidean norm of the corresponding vector; $q$ is a set constant, and $q>0$ reflects the tracking rate of $\hat{w}_{\mathrm{p}}$ to $\boldsymbol{w}_{p}$, the bigger $q$ is, the faster tracking rate of $\hat{w}_{\mathrm{p}}$ to $\boldsymbol{w}_{p}$ is. Obviously, $V(t)>0$.

Taking derivative of both sides of Formula (9) and we can get that,

$$
\frac{\mathrm{d} V(t)}{\mathrm{d} t}=\boldsymbol{z}_{\boldsymbol{e}}^{T} \boldsymbol{N} \frac{\boldsymbol{d} z_{e}}{\boldsymbol{d} t}+\frac{1}{2 q}\left[\left\|\boldsymbol{w}_{\boldsymbol{p}}(t)-\hat{\boldsymbol{w}}_{\boldsymbol{p}}(t)\right\|^{2}-\left\|\boldsymbol{w}_{\boldsymbol{p}}\left(t-T_{f}\right)-\hat{\boldsymbol{w}}_{\mathrm{p}}\left(t-T_{f}\right)\right\|^{2}\right]
$$

Handling the first item at the right side of equal mark in Formula (10) and taking Formula (8) into account, we can get that,

$$
\boldsymbol{z}_{e}^{\mathrm{T}} \boldsymbol{N} \frac{\mathrm{d} z_{e}}{\mathrm{~d} t}=\boldsymbol{z}_{e}^{\mathrm{T}}\left[-\boldsymbol{J} \boldsymbol{z}_{e}-\boldsymbol{R} \boldsymbol{z}_{e}+\boldsymbol{\xi}+\hat{\boldsymbol{w}}_{\mathrm{p}}(t)+\boldsymbol{w}_{1}\right]+\boldsymbol{z}_{e}^{\mathrm{T}}\left[\boldsymbol{w}_{\mathrm{p}}(t)-\hat{\boldsymbol{w}}_{\mathrm{p}}(t)\right]
$$

Because $\boldsymbol{w}_{p}(t)=\boldsymbol{w}_{p}\left(t-T_{f}\right)$, then the $2^{\text {nd }}$ item on the right of equal mark in Formula (10) can be also simplified, and we can get that,

$$
\begin{aligned}
& \left(\left\|\boldsymbol{w}_{\boldsymbol{p}}(t)-\hat{\boldsymbol{w}}_{\boldsymbol{p}}(t)\right\|^{2}-\left\|\boldsymbol{w}_{\boldsymbol{p}}\left(t-T_{f}\right)-\hat{\boldsymbol{w}}_{\boldsymbol{p}}\left(t-T_{f}\right)\right\|^{2}\right) \\
& =2\left(\hat{\boldsymbol{w}}_{\boldsymbol{p}}\left(t-T_{f}\right)-\hat{\boldsymbol{w}}_{\boldsymbol{p}}(t)\right)^{\mathbf{T}}\left(\boldsymbol{w}_{\boldsymbol{p}}(t)-\hat{\boldsymbol{w}}_{\boldsymbol{p}}(t)\right)-\left\|\hat{\boldsymbol{w}}_{\boldsymbol{p}}\left(t-T_{f}\right)-\hat{\boldsymbol{w}}_{\boldsymbol{p}}(t)\right\|^{2}
\end{aligned}
$$

Based on the repetitive control of internal model principle, to make gradual pointed compensation by use of the periodic law of error signal, can eliminate all periodic errors within closed-loop system, gradually reduce or eliminate systemic periodic errors, thus make system output achieve expected objective. The structure of repetitive control is simple and easy to realize, which provides the compensation of periodic disturbance with a very valuable approach. In order to compensate periodic disturbance $\boldsymbol{w}_{\boldsymbol{p}}$, define the estimated vector of $\boldsymbol{w}_{p}-\hat{\boldsymbol{w}}_{p}$ by use of repetitive control as below,

$$
\hat{\boldsymbol{w}}_{\boldsymbol{p}}(t)=\hat{\boldsymbol{w}}_{\boldsymbol{p}}\left(t-T_{f}\right)+q z_{\boldsymbol{e}}
$$

Substitute Formula (11) to Formula (13) into Formula (10) and get that,

$$
\frac{\mathrm{d} V(t)}{\mathrm{d} t} \leq \boldsymbol{z}_{e}^{\mathrm{T}}\left(-\boldsymbol{J} z_{e}-\boldsymbol{R} \boldsymbol{z}_{e}+\boldsymbol{\xi}+\hat{\boldsymbol{w}}_{\mathrm{p}}(t)+\boldsymbol{w}_{1}\right)
$$

Carry out equivalence transformation for $z e^{T} w l$ and get that,

$$
\boldsymbol{z}_{\mathrm{e}}^{\mathrm{T}} \boldsymbol{w}_{1} \leq \frac{r^{2}\left\|\boldsymbol{w}_{1}\right\|^{2}-\left\|\boldsymbol{z}_{e}\right\|^{2}}{2}+\boldsymbol{z}_{e}^{\mathrm{T}}\left(\frac{1}{2}+\frac{1}{2 r^{2}}\right) \boldsymbol{z}_{e}
$$

In the formula, $r>0$ denotes the interference suppression standard of error system, the smaller $r$ is, the smaller deviation degree of error system state from the equilibrium point is, as for the same disturbance.

Substitute Formula (15) into Formula (14), because $z_{e} \boldsymbol{T}_{\boldsymbol{T}} \boldsymbol{z}=0$, we can get that, 


$$
\frac{\mathrm{d} V(t)}{\mathrm{d} t} \leq-\boldsymbol{z}_{e}^{\mathrm{T}} \boldsymbol{R} \boldsymbol{z}_{e}+\frac{r^{2}\left\|\boldsymbol{w}_{1}\right\|^{2}-\left\|\boldsymbol{z}_{e}\right\|^{2}}{2}+\boldsymbol{z}_{e}^{\mathrm{T}}\left[\boldsymbol{\xi}+\hat{\boldsymbol{w}}_{\mathrm{p}}(t)+\left(\frac{1}{2}+\frac{1}{2 r^{2}}\right) \boldsymbol{z}_{e}\right]
$$

Passive control theory is an important tool to design robust equanimity controller in nonlinear system, which is a kind of control method for energy shaping. By the infusion of nonlinear damping, it can compel the total energy of system to track the expected energy function, force the systematic state variable to asymptotically converge to the set value, and attain the performance required by system. The physical significance of passive control is to indicate that the increment of systematic energy from initial moment to current moment is always less than or equal to the sum of energy infusing from outside into the system, and the movement of passive system is always accompanied by the loss of energy. This control method has overall definition and is stable in whole without singular point, with simple design of corresponding controller, easy realization in reality and strong robustness.

As for the Formula (16), by the use of passive control principle, infuse damping via dynamic feedback and let

$$
\xi+\hat{w}_{p}(t)+\left(\frac{1}{2}+\frac{1}{2 r^{2}}\right) z_{e}=-R_{d} z_{e}
$$

The concrete form of $\boldsymbol{R}_{\mathrm{d}}$ is $\boldsymbol{R}_{\mathrm{d}}=\operatorname{diag}\left[r_{d a}, r_{d b}, r_{d c}, r_{d d}\right]$, among which $r_{d a}, r_{d b}, r_{d c}, r_{d d}$ are damping coefficients and $r_{d a} \geq 0, r_{d b} \geq 0, r_{d c} \geq 0, r_{d d} \geq 0$.

Substitute Formula (17) into Formula (16) and get that,

$$
\frac{\mathrm{d} V(t)}{\mathrm{d} t} \leq-\boldsymbol{z}_{e}^{\boldsymbol{T}}\left(\boldsymbol{R}+\boldsymbol{R}_{\mathrm{d}}\right) \boldsymbol{z}_{e}+\frac{r^{2}\left\|\boldsymbol{w}_{l}\right\|^{2}-\left\|\boldsymbol{z}_{e}\right\|^{2}}{2}
$$

Define $z=\operatorname{diag}(1,1,1,1)$, and $z e$ is the evaluation signal to evaluate the performance of Error System Formula (8). Obviously, when $\boldsymbol{w} l=0$, Error System (8) is detectable under zero state for $\boldsymbol{z}$. Meanwhile, we can know from $-z_{e}{ }^{T}\left(\boldsymbol{R}+\boldsymbol{R}_{\mathrm{d}}\right) \boldsymbol{z}_{e} \leq 0$ and Formula (18) that, $z_{e}=0$ is the robust asymptotic stable equilibrium point of Error System Formula (8), namely, to realize the design of robust $L_{2}$ performance criteria for Error System Formula (8) via Formula (13) and Formula (17).

By association between Formula (14) and Formula (18) and to let $k_{r d x}=\frac{1}{2}+\frac{1}{2 r^{2}}+r_{d x}>0.5$, we can get that, the controller of error system model of Formula (8) is:

$$
\left\{\begin{array}{l}
p_{x}=\left[u_{s x}-\left(L \frac{\mathrm{d} i_{c x}^{*}}{\mathrm{~d} t}+R i_{c x}^{*}\right)+\hat{w}_{\mathrm{px}}(t)+k_{r d x}\left(i_{c x}-i_{c x}^{*}\right)\right] / u_{\mathrm{d}}^{*} \\
-\left(C \frac{\mathrm{d} u_{d}^{*}}{\mathrm{~d} t}-p_{a} i_{c a}^{*}-p_{b} i_{c b}^{*}-p_{c} i_{c c}^{*}+Y_{\mathrm{d}} u_{\mathrm{d}}^{*}\right)+\hat{w}_{p d}(t)+k_{r d x}\left(u_{\mathrm{d}}-u_{\mathrm{d}}^{*}\right)=0 \\
\hat{w}_{p x}(t)=\hat{w}_{p x}\left(t-T_{f}\right)+q\left(i_{c x}-i_{c x}^{*}\right) \\
\hat{w}_{p d}(t)=\hat{w}_{p d}\left(t-T_{f}\right)+q\left(u_{\mathrm{d}}-u_{\mathrm{d}}^{*}\right)
\end{array}\right.
$$

In theory, so long as the reference value of state is set, under the function of Robust Current Controller (19) based on the combination between repetitive control and passive control, system state will be always asymptotically stable to the reference value. Then, Error System Model (8) has 4 independent quantities of state $z_{e k}(k=a, b, c, d)$, but only 3 controlled quantities $p_{x}(x=a, b, c)$, because this system is under actuated nonlinear dynamic system. By use of indirect control method, this paper can give $i_{c x}{ }^{*}$ an active current component via PI control, and realize control of stable DC voltage, namely, realize $u_{d} \rightarrow u_{d}{ }^{*}$, at the same time of controlling $i_{\mathrm{cx}} \rightarrow i_{c x}{ }^{*}$ to realize harmonic current control.

\section{Simulation \& engineering application}

\subsection{Simulation result}

In order to verify the accuracy of the above mathematical model and control the validity of algorithm, by use of Matlab/Simulink, this paper builds a simulation model with main circuit topology and $380 \mathrm{~V}$ of voltage class as shown in Fig.1. The simulation parameters are shown as Table 1, among which $f_{0}$ is the switching frequency of IGBT. 
Table 1. Simulation Parameter

\begin{tabular}{cc|cc}
\hline Parameter & Value & Parameter & Value \\
\hline$L / \mathrm{mH}$ & 0.3 & $C / \mu F$ & 4700 \\
& 0.35 & $C d / \mu F$ & 4200 \\
$R / \Omega$ & 0.0015 & $(1 / Y) / \Omega$ & $<3000$ \\
$/ \Omega$ & $>0.002$ & $\left(1 / Y_{d}\right) / \Omega$ & $<2000$ \\
$/ \mathrm{V}$ & 800 & $f_{0} / \mathrm{Hz}$ & 9600 \\
\hline
\end{tabular}

Fig. 2 shows A-phase load current waveform when the system carries three-phase uncontrolled rectifier bridge load. As shown in the figure, the waveform distortion of load current is serious, and the total harmonic distortion $>25 \%$. Fig.3 is A-phase current waveform at the side of system after APF device begins to work. The current waveform distortion is obviously improved, and the distortion factor drops to be less than $4 \%$.

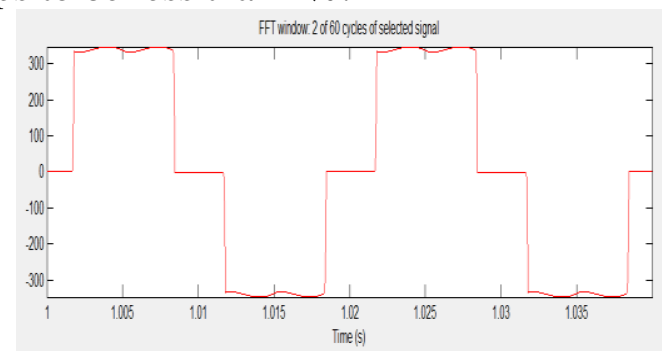

(a) Current waveform

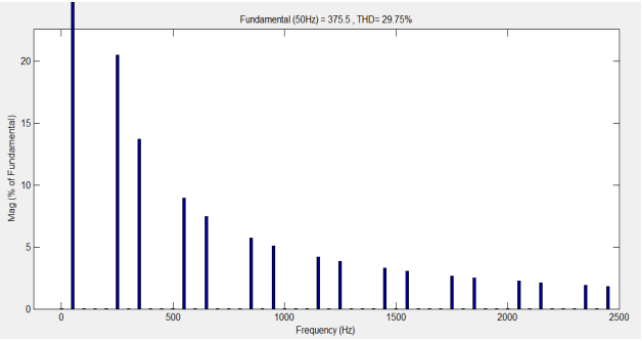

(b) Spectrum waveform

Fig. 2 Load current waveform and spectrum waveform

Fig.4 shows A-phase directive current $i_{a}{ }^{*}$ and offset current $i_{c a}$ waveforms of the device under the function of robust current control. As shown in the figure, under the function of robust current control, the offset current can realize tracking without static error of the directive current.

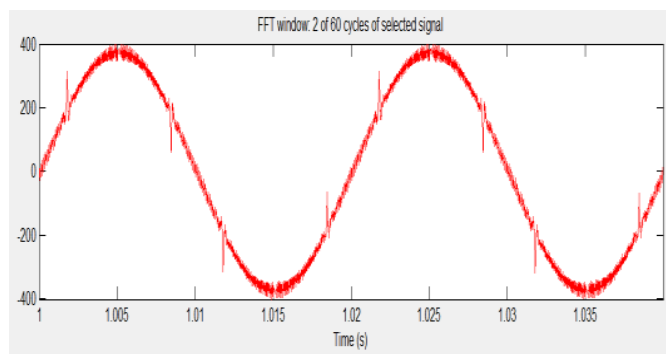

(a) Current waveform

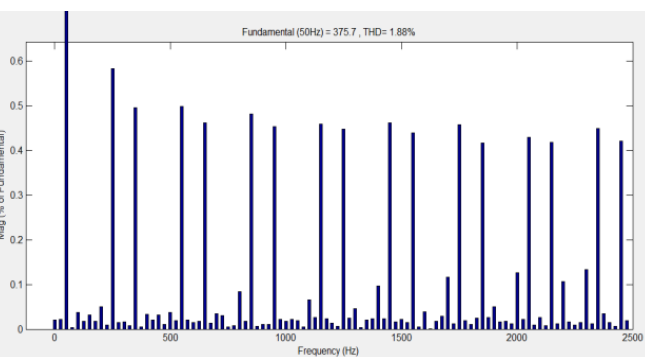

(b) Spectrum waveform

Fig. 3 System current waveform under the robust current control

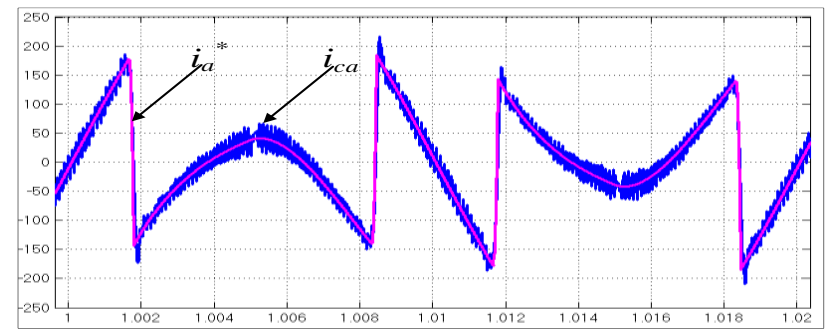

Fig. 4 The waveform of the reference current and the compensation current under the robust current control

Fig.5 shows the waveforms of A-phase directive current and offset current under the individual function of passive control. As shown in Fig.5, under the passive control, directive current and offset current have certain static errors. 


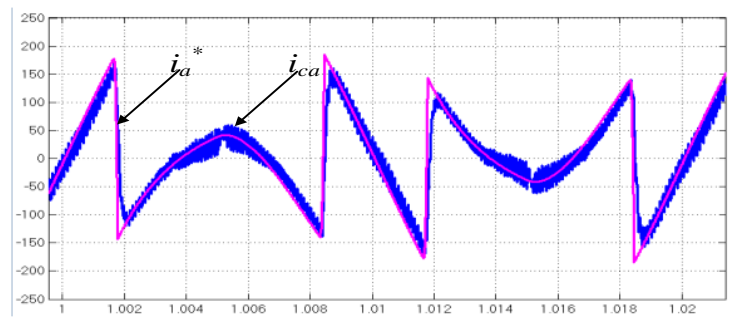

Fig. 5 The waveform of the reference current and the compensation current under the passivity control

\subsection{Engineering application verification}

The control algorithm mentioned in this paper is verified in APF device used for industry. The class of system voltage is $380 \mathrm{~V}$, the rated value of DC voltage is $800 \mathrm{~V}$, the capacitance at the side of capacitor is $4700 \mu \mathrm{F}$, and the switching frequency of IGBT is $9600 \mathrm{~Hz}$. The controller consists of DSP and FPGA. DSP is used to realize the effective values in calculation, the system-level protection, the running process of device and so on. FPGA realizes the detection of harmonic current, the control of robust current, the control of DC Voltage and so on. The touch screen uses RS485 hardware interface and Modus RTU communication protocol to realize the data exchange with DSP.

Fig.6 is the systematic current waveform before the commissioning of device, and Fig.7 is the systematic current waveform of device after commissioning. By the analysis of experimental results, we can know that the total distortion factor of system current before commissioning is about $25 \%$, and after commissioning, the total distortion factor of system current is less than $5 \%$.

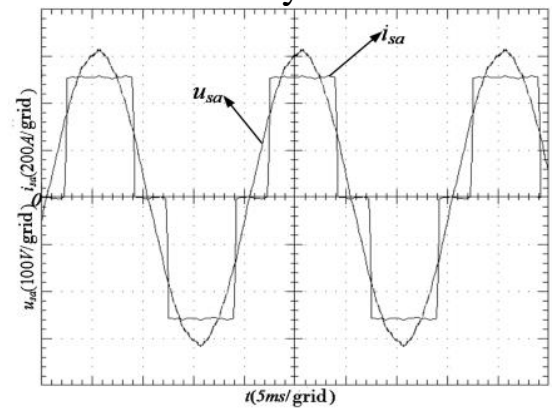

Fig.6 Waveforms of system voltage, system current before compensation

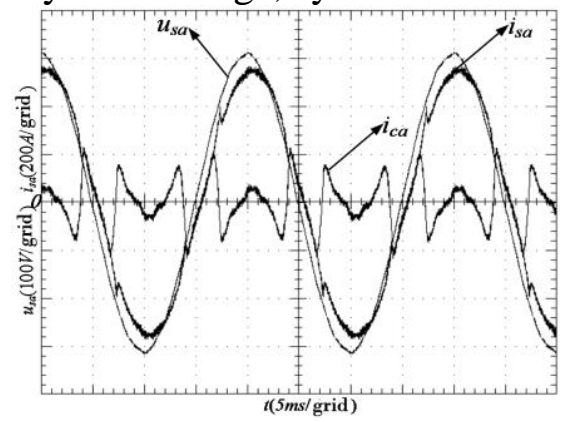

Fig.7 Waveforms of system voltage, system current and compensation current

\section{Conclusion}

The operation condition of active power filter is very complex. This paper firstly establishes the precise mathematical model of three-phase shunt active power filter in $a b c$ coordinate system under parameter perturbation and unknown disturbance in complex operating condition; By the equivalence transformation of the model, it converts the influence of parameter perturbation to a part of periodic disturbance; by the new method combining repetitive control and passive control, it realizes the robust current controller design of APF. Both simulation and engineering application results can control the total distortion factor of system current to be less than $2 \%$, which verifies the precision and the validity of control algorithm of this mathematical model, with high theoretical significance and practical application value, and can be used by other power electronic converter topology for reference. 


\section{References}

[1] Wang Zhao'an, Yang Jun, Liu Jinjun. Harmonic Suppression \& Reactive Power Compensation [M]. Beijing: China Machine Press, 1998: 1-364.

[2] Fan Ruixiang, Luo An, Tang Jie. Delay Research on Digital Control System with Harmonic Wave Injection Type of Active Power Filter [J]. Journal of Chinese Electrical Engineering Science, 2007,27(13):104-110.

[3] He Na, Wu Jian, Xu Dianguo. Fuzzy Control on DC Voltage of Active Electric Power Filter [J]. Power Grid Technology, 2006, 30(14):45-48.

[4] Zhou Lin, Jiang Jianwen, Zhou Luowei, et al. Active Electric Power Filter of Three-phase Four-wire System Based on One-cycle Control [J]. Journal of Chinese Electrical Engineering Science, 2003, 23(3):85-88.

[5] He Yuqu, Yan Maode. Nonlinear Control Theory \& Application [M]. Xi'an: Xidian University Press, 2007:105-190.

[6] Xu Jianxin. A new periodic adaptive control approach for time-varying parameters with know periodicity [J]. IEEE Trans. on Automatic Control,2004 49[4]:579-583

[7] Zhang Zhenhuan, Liu Huijin. New Method of Gain Repetitive Control for One-phase Active Electric Filter Journal of Chinese Electrical Engineering Science, 2008,28(12):79-87.

[8] Gao Jun, Li Hui, Yang Xu, et al. Research on Sine-wave Inverter Power Source Based on PID Control and Repetitive Control [J]. Advanced Technology of Electrical Engineering and Energy, 2002, 21(1):1-4.

[9] Zhang Zhenhuan, Liu Huijin, Li Qionglin, et al. New Method of Passive Control for One-phase Active Electric Filter Based on Euler-Lagrange Model [J]. Journal of Chinese Electrical Engineering Science, 2008, 28(12):79-87.

[10]Wang Jiuhe, Huang Lipei, Yang Xiuyuan, Passive Power Control of PWM Rectifier of Three-phase Voltage Type [J]. Journal of Chinese Electrical Engineering Science, 2008, 28(21):20-25.

[11] Wang Jiuhe. Passive Control Theory and Its Application [M]. Beijing: Electronic Industry Press, 2010.

[12]Zhang Maosong, Li Shangsheng, Zha Xiaoming, et al. Robust Performance Criteria Design of Chained D-STATCOM [J]. Journal of Chinese Electrical Engineering Science, 2011, 31(28):71-79.

[13]Zhang Maosong, Wang Qunjing, et al. Research on Robust Current \& Midpoint Potential Control Strategy of Terminal Point Clamping Type of Tri-level D-STATCOM [J]. Journal of Chinese Electrical Engineering Science, 2016, 36(14):68-77.

[14] Mei Shengwei, Shen Tielong, Liu Kangzhi. Modern Robust Control Theory \& Application [M]. Beijing: Tsinghua University Press, 2008:194-197.

[15] About the Author:

[16] Shu Zhan (1977-), male, is a master and a senior engineer. And the research method is electric system automation. 